\title{
Krise der Abonnentenwerbung in der konfessionellen Presse
}

\author{
von Ferdinand Oertel
}

Im Mittelpunkt der gegenwärtigen Überlegungen zur Stabilisierung und Verbesserung der katholischen Presse in der Bundesrepublik stehen bei der Bischofskonferenz, der Synode und dem Zentralkomitee der Deutschen Katholiken Pläne für die Schaffung leistungsfähiger Organe. Ein Ausbau der Katholischen Nachrichtenagentur KNA, eine Verstärkung der Einzelredaktionen, vermehrte Kooperation zwischen einzelnen Bistums- und Missionsblättern, schließlich eine - wie immer auch geartete - Gemeinschafts- oder Kernredaktion der Kirchenpresse haben alle dasselbe Ziel: bessere Zeitschriften zu ermöglichen.

Dieses Ziel ist bestimmt erstrebenswert und innerhalb eines publizistischen Gesamtplanes der Kirche in der Bundesrepublik auch vorrangig. Aber man sollte sich darüber klar werden, daß es mit der Erstellung leistungsfähigerer, besserer, offenerer Blätter noch längst nicht getan ist, im Gegenteil: Eine noch so gut gemachte Zeitschrift verfehlt ihre Aufgabe, wenn sie nicht ihren Leserkreis findet. Auch noch so hohe finanzielle Subventionen helfen letzten Endes nichts, wenn nicht eine wesentliche Funktion gesichert ist: die gezielte Heranführung des Objektes an den intendierten Leserkreis. Mit anderen Worten: die Abonnentenwerbung. Und gerade diese entscheidende Frage wird noch kaum erörtert, obwohl sie auch im Fall "Publik" eine wesentliche Rolle gespielt hat.

\section{Lebren aus der Werbung für „Publik ${ }^{\star}$}

In der "Information"1, die zur Geschichte von „Publik" von einer gemischten Kommission für die Synode erstellt worden ist, wird auf diese besondere Werbesituation hingewiesen. Bei "Publik“ wurde demnach anfangs alles darangesetzt, um das Image einer ferngesteuerten „Bischofszeitung“ gar nicht erst aufkommen zu lassen: „So wollte man zunächst den Weg über Empfehlungen durch Pfarrer und kirchliche Verbände nicht gehen; die Zeitung sollte sich durch ihre publizistische Leistung rasch genug auf dem freien Markt durchsetzen. Hier konnten also Werbechancen im Einzugsgebiet der Kirche nicht genutzt werden."2 Sie sollten - hätte es sie gegeben auch gar nicht genutzt werden, denn "Publik“ wollte sich bewußt von der Kirchenpresse abheben und auch auf dem innerkirchlichen Werbemarkt keine Abwerbung betreiben. Als sich schon bald herausstellte, daß die Zeitschrift auf dem freien Markt nicht die erhoffte Auflage erreichte, ging man dann doch zu der bei allen anderen kirchlichen Presseorganen üblichen Abonnentenwerbung über: der Kolonnenwerbung. Hierbei mußte man jedoch sehr bald eine Erfahrung machen, die den Verlegern der Kirchenpresse nicht neu ist: Die Kolonnenwerbung ist mit einer hohen Leserfluktuation verbunden. Die "Information" führt so auch an, daß 1970 bei 52000 Neuzugängen 24000 Abgänge zu verzeichnen waren, 1971 bei 56000 Neuzugängen sogar 54000

Dr. Ferdinand Oertel ist Chefredakteur der Wochenzeitung „Die christliche Familie“ und Vorsitzender der Arbeitsgemeinschaft Katholische Presse. Seinen für CS verfaßten Beitrag nahm der Verfasser in ein Taschenbuch auf, das im Herbst 1972 unter dem Titel "Sprachrohr oder Dialogforum?" im Lahn-Verlag, Limburg, erscheinen wird. 
Abgänge, d. h. eine Fluktuation von 95 Prozent. Als Grund für das Scheitern dieser außerordentlichen Werbeanstrengungen durch Kolonnenwerbung an den Haustüren wird in der "Information “ angegeben, daß diese Werbung "der redaktionellen Konzeption des Blattes nicht entsprach“.

Es ist müßig, an dieser Stelle Erwägungen darüber anzustellen, ob die Werbung für "Publik“ auf dem freien Markt, der demnach offenbar der redaktionellen Linie entsprochen haben muß, über einen längeren Zeitraum doch zu der tragfähigen Auflage geführt hätte. Die Erfahrungen bei der Werbung für „Publik" haben jedoch - und darauf kommt es hier an - klar erwiesen, daß die Abonnentenwerbung für katholische Zeitschriften anders gelagert ist als für andere politische Wochenzeitungen, für die Illustrierten und alle sonstigen Publikumszeitschriften. Diese werden vorwiegend am Kiosk verkauft.

\section{Empfeblung vom Pfarrer?}

Für den Kiosk-Verkauf kann mit Plakaten, Zeitungsanzeigen oder Spots in Funk und Fernsehen geworben werden, ohne daß genaue Angaben über Bezugsmöglichkeiten gemacht werden müssen; denn die betreffenden Publikationen sind unmittelbar an jedem Kiosk an der nächsten Ecke zu kaufen. Die katholische Presse ist auf diese Weise aber nicht zu haben. Früher gab es die Illustrierte „Feuerreiter" zwar am Kiosk, jetzt ist dort gelegentlich noch "Weltbild“ zu finden, und „Publik" hat es auch am Kiosk gegeben, aber die Verkaufszahlen lagen dort weit unter der Rentabilität. Kioskaushang hat für diese Zeitschriften höchstens einen begrenzten Charakter als Image-Pflege. Als wirtschaftlicher Verkaufsträger kommt der Kiosk für die konfessionelle Presse insgesamt nicht infrage.

Die konfessionelle Presse ist in der Hauptsache auf die Türwerbung angewiesen. Diese wird von den Werbekolonnen durchgeführt. Früher war die Situation für diese Türwerbung wesentlich besser als heute. Die meisten Pfarrbüros nahmen sowieso den Vertrieb vieler katholischer Blätter vor. Das ist heute längst nicht mehr überall der Fall, weil die Pfarrbüros anderweitig überlastet sind, weil der Vertrieb nichts einbringt oder man auf diese Einnahmen nicht mehr angewiesen ist - wie etwa in Italien, wo die Wochenillustrierte "Famiglia cristiana" u. a. deshalb eine Auflage von 1,8 Millionen verkaufen kann, weil der Vertrieb für die italienischen Geistlichen einen willkommenen Nebenerwerb bietet. Die katholischen Pfarrbüros halfen früher aber auch ohne weiteres bei der Werbung, indem sie den Werbern Empfehlungen für die katholischen Blätter gaben. Die Werber kamen dann „mit einem schönen Gruß vom Herrn Pastor" in die Häuser und fanden offene Türen vor, was sich meistens in einem Abonnement auszahlte, zumal man etwas "für die gute Sache“ tat und es nie schadete, wenn der Herr Pastor davon Kenntnis nahm. Heute gibt es solche Empfehlungen von Pfarrern nur noch für die Bistumsblätter. Dadurch wird die Situation der anderen, vorwiegend privatwirtschaftlich herausgegebenen katholischen Blätter doppelt schwer. Doch auch mit einer Empfehlung von Pfarrern finden die Werber der Bistumspresse heute nicht mehr so viele offene Türen vor wie noch vor einem Jahrzehnt.

Für die anderen katholischen Pressegruppen hat sich die Situation ebenfalls erschwert. $\mathrm{Da}$ das Verbandsleben längst nicht mehr so ausgeprägt ist wie früher, fallen diese Organisationen auch als Werbeträger ganz oder fast ganz aus. Bestes Beispiel dafür ist die katholische Jugend, von deren blühender Presse der 50er Jahre nichts mehr 
übriggeblieben ist. Ein weiteres Beispiel ist die Illustrierte „Weltbild“, die aus dem „Feuerreiter " und der katholischen Männerzeitschrift "Mann in der Zeit " entstanden ist. Vom Männerverband allein kann diese Zeitschrift heute nicht mehr getragen werden. Wo hingegen Blätter als Entgelt für die Mitgliedschaft geliefert werden, sieht die Situation anders aus. Seit das „Kolpingblatt“ seinen Bezug für die Mitglieder verpflichtend gemacht hat, ist die Auflage stabiler geworden. Bei vielen Ordens- und Missionszeitschriften, die ebenfalls als Gegenleistung für Beiträge oder Spenden geliefert werden, sinkt jedoch die Auflage, weil offensichtlich das Interesse am Missionsgedanken stark nachläßt.

\section{Türwerbung obne Zukunft}

Grundsätzlich bleibt die katholische Presse also auf die Türwerbung angewiesen. Doch auch diese wird immer problematischer. Der Kauf an der Haustüre ist vom Odium des Betrugs belastet, und in der Tat wenden die Türwerber vielfach Methoden an, die fragwürdig sind. Für die Objekte selbst, die einzelnen Zeitschriften, wird oft gar nicht direkt geworben. Es geht den Werbern vielmehr darum, um jeden Preis einen "Schein" zu schreiben. Deshalb verschaffen sie sich mit allen möglichen Tricks Eingang in die Wohnungen, indem sie etwa behaupten, eine Aktion gegen Schmutz und Schund durchzuführen (bei der es dann auch eine Zeitschrift als Zutat gibt), oder zur Unterstützung von Krankenhäusern aufrufen (was durch ein Patenschaftsabonnement geschehen kann) usw.

Viele Werbekolonnen wandern von Verlag zu Verlag und werben dann an Hand ihrer Karteien die Bezieher jeweils für ein anderes Blatt um, wenn die Verpflichtungszeit von einem oder eineinhalb Jahren abgelaufen ist. Noch so gute redaktionelle Leistungen bewirken dann keine Verlängerung der Leserbindung, weil für die Zeitschriften unter falscher Flagge geworben wurde und die Bezieher dadurch psychologisch gegen das Blatt voreingenommen sind. Alle Versuche der Verleger konfessioneller Zeitschriften - die evangelische Presse befindet sich in derselben Werbesituation -, eine Art Ehrenkodex für die Kolonnen verpflichtend zu machen, scheitern an der harten Realität auf dem Markt der "Türdrücker".

Es hat wenig Sinn, die Augen vor diesen Tatsachen zu verschließen und zu hoffen, daß in Zukunft mit solcher Türwerbung noch große Erfolge erzielt werden können. Rechnete man noch vor zehn Jahren mit höchstens 40 Prozent "Springern“, d.h. Rückgängen unter den Türabonnements, so liegt diese Zahl heute zwischen 90 und 100 Prozent. Wenn es erst ein gesetzliches Rücktrittsrecht von Kaufverträgen an der Tür gibt, wird die Situation noch ungünstiger.

Die Kosten für die Werbung liegen so hoch, daß ein neuer Bezieher die Zeitschrift schon jetzt weit über den Verpflichtungszeitraum hinaus beziehen muß, ehe der Werbeaufwand gedeckt ist und der Bezug Erlös bringt. Auch die Bestrebungen, um jeden Preis neue Abonnenten zu werben, um die Auflage zu halten, weil dann wenigstens gewisse Anzeigenaufträge bleiben, zahlt sich auf die Dauer nicht aus. Diese Werbekosten sind so hoch, daß sie an die Substanz der Blätter gehen.

\section{Werbekonzept für die Gemeinden}

Deshalb ist es im Rahmen der Überlegungen für ein Gesamtkonzept der kirchlichen Publizistik höchste Zeit, ein Werbekonzept zu entwerfen, das aus dieser tödlichen 
Situation herausführt. Ein solches Konzept kann nur dort ansetzen, wo die katholischen Blätter gelesen werden sollen: in den Gemeinden. Wenn dort kein Verhältnis zur eigenen Presse gefunden wird, ist diese zum Untergang verurteilt. Katholische Presse wird immer in der Hauptsache von Katholiken gelesen, die sich noch zur Kirche bekennen. Die Absicht, mit einem Blatt „aus dem katholischen Ghetto“ heraus die Rand- und Nichtchristen ansprechen zu wollen, wäre falscher Ehrgeiz. Das kann vielleicht durch andere Medien geschehen. Katholische Presse muß sich an die bekennenden Katholiken wenden, was im übrigen keinesfalls zu heißen braucht, daß sie deshalb ghettohaft gestaltet sein müßte. Einerseits leben heute nur noch wenige Katholiken in einem Ghetto - sofern das in unserer pluralen Gesellschaft überhaupt noch möglich ist -, andererseits gibt es unter den Katholiken verbindende Elemente der Lebenserwartungen und -haltungen, die eine zielbewußte Gesinnungspresse durchaus rechtfertigen, ganz zu schweigen vom pastoralen Auftrag, der nicht nur Elitegruppen umfaßt.

Das Leserpublikum für die katholische Presse wird schwerpunktmäßig in jenen Schichten zu finden sein, die auf die Umfrage unter den Katholiken zur Vorbereitung der Synode geantwortet haben. Es ist in etwa auch identisch mit der Zahl der aktiven Katholiken in den Gemeinden. Und wo es darum geht, Randchristen und Nichtchristen anzusprechen, muß auch dies im Wesentlichen an der Basis der Kirche geschehen; das Lesen einer "fortschrittlichen" Zeitschrift allein dürfte kaum Impulse für eine Glaubensaktivierung wecken.

Gerade im Zuge der Konzilserneuerung hat die pastorale Belebung der Gemeinden besondere Bedeutung bekommen. Tragende Elemente für jede Gemeindearbeit sind Information, Kommunikation, Kontakte und gemeinsame Aktivitäten. Dabei werden in den nächsten Jahren die Medien eine immer stärkere Rolle spielen. Hier liegt die Zukunftschance der katholischen Presse. Ihr tragfähiges Reservoir liegt in der aktiven Gemeinde.

Die katholische Presse wird ihrerseits eine Spezialisierung erfahren müssen; denn jedes Blatt kann nicht mehr allen alles bieten wollen. Die Bistumspresse dürfte ihren Schwerpunkt im Forumscharakter für das Bistum finden, andere Blätter werden ergänzende Bereiche der Familie, Erziehung, Jugend, Entwicklungshilfe und Mission behandeln können. Es ist fraglich, ob dann noch Platz für jene 160 Titel da ist, die es heute noch gibt. Sie alle hatten ihre Berechtigung zu ihrer Zeit und aus ihrer Aufgabenstellung, zum Teil aber haben sie ihre Funktion verloren und können auch durch Subventionen nicht mehr lange am Leben gehalten werden. Aber fraglos ist, $\mathrm{da} ß$ in den Gemeinden und beim einzelnen Katholiken Bedarf an katholischer Presse bestehen bleibt, die in den einzelnen Bereichen des gemeindlichen- und bistumsbezogenen Glaubenslebens ihre informierende, kommunikative und pastorale Rolle behält.

Wenn in Zukunft die Gemeinden zu den Trägern der katholischen Presse werden müssen, dann ist dort ein Umdenken und Umlernen erforderlich. Es muß derselbe Prozeß in Gang kommen, mit dem das Konzil eine neue Haltung gegenüber allen Medien eingeleitet hat (vgl. die konziliaren Dokumente Inter Mirifica und Communio et progressio). Speziell jene Presse, die als katholische Presse in der Verantwortung der Kirche erscheint, muß in ihrer gewandelten Funktion gesehen werden. Sie ist nicht mehr Sprachrohr von oben, sondern sie erfüllt eine kommunikative Funktion in Gemeinde, Bistum und Gesamtkirche. Von daher sind Gemeinden und 
Bistümer auch in erster Linie verantwortlich für die Kirchenpresse. Von daher kommt die Verpflichtung zur direkten Förderung.

\section{Ein katholisches Vertriebs- und Werbenetz?}

Hat eine solche Wandlung in der Haltung gegenüber der katholischen Presse stattgefunden, dann kommen gerade die Gemeinden als die neuen Werbeträger für die Kirchenpresse infrage. Dann weiß man dort, wer welches Blatt wirklich braucht. Die direkte "Werbung “ könnte zunächst nur nebenamtlich beginnen, müßte aber im Endziel $\mathrm{zu}$ einer festen Institution führen. In der Arbeitsgemeinschaft Katholische Presse ist in jüngster Zeit der Plan einer katholischen Vertriebs- und Werbegemeinschaft angeregt worden. Er bedarf gewiß noch vieler Einzelüberlegungen. Noch scheinen z. B. die Bahnen der konkurrierenden Verlage nicht so parallel zu verlaufen, daß ein solches Netz gemeinsamer Vertriebs- und Werbestellen in jeder Pfarrei aufgerichtet werden könnte. Noch beanspruchen die Bistumsblätter den Vorrang unter allen katholischen Blättern; was sich ändern würde, wenn sie zum spezifischen Dialogforum der Ortskirche entwickelt würden. Ungelöst ist das Problem, ob für verschiedene Objekte gemeinsam geworben werden kann, unklar ist, ob beim gemeinsamen Kassieren der Bezugsgelder für mehrere Objekte die Bezieher nicht dazu verführt werden, infolge der ihnen bewußt werdenden Gesamtsumme einzelne Blätter abzubestellen.

Die Sondersituation einer Gesinnungspresse auf dem freien Wirtschaftsmarkt wird jedoch sehr bald die Kirche (die Kirche und nicht die Verleger!) aus pastoralen Gründen zwingen, an der Basis - in den Gemeinden und Gemeindeverbänden eine neue Trägerschaft für ihre Presse zu schaffen. Und jede Starthilfe, die dafür gewährt würde, oder jede finanzielle Mitbeteiligung wäre besser angelegt als zur Förderung einzelner Organe. Eine solche Vertriebs- und Werbegesellschaft, die im übrigen auch für die Verbreitung audiovisueller Medien in Betracht käme, muß als eigenes Wirtschaftsunternehmen gestartet werden. Die örtlichen Agenturen müssen und können - entsprechend finanziert werden. Die Kirche, vertreten durch die Bischofskonferenz, könnte im übrigen den katholischen Verlegern sogar den Anreiz einer Finanzhilfe bieten, um sie rascher zur Gründung einer solchen Einrichtung zu bewegen. Dieser Plan ist jedenfalls konkreter als viele andere Projekte.

Ein abgerundetes Gesamtkonzept für die kirchliche Publizistik wird immer Utopie bleiben, was nicht die Erarbeitung von dringlichen Einzelprojekten in größerer $\mathrm{Zu}$ sammenschau ausschließt. Was eher zu verwirklichen wäre, ist ein Konzept für publizistische Arbeit in jedem Bistum. Und dieses Konzept dürfte nicht bei den Medien, nicht bei der Einrichtung von Pressestellen, Pressesprechern, audiovisuellen Zentren und sonstigen Institutionen beginnen, sondern es müßte in den Gemeinden grundgelegt werden. Wenn die Kommunikationsbedürfnisse an der Basis erkannt sind, kann auch keine Zeitung mehr am Publikum vorbeigemacht werden. Es entstünde eine fruchtbare Wechselbeziehung zwischen denjenigen, die katholische Presse herausgeben, denjenigen, die sie machen, und denjenigen, für die sie gemacht werden.

\section{Anmerkungen:}

1. Gemeinsame Synode der Bistümer in der Bundesrepublik Deutschland: Information zu ,Publik', vorgelegt von der gemischten Kommission „Grundsätze für ein Gesamtkonzept kirchlicher Publizistik", 33 Seiten und 2 Anlagen.

2. A.a.O., S. 22. 


\section{SUM MAR Y}

Despite all other attempts to improve the Catholic Press in Germany, the direct work of obtaining funds is often overlooked. Apart from political weeklies, illustrated and other papers for the general public, the professional Press depends largely on advertising and sales from door to door. This method is becoming more and more difficult today. Advertisers at the door advertise everything. They must obtain a transient success by every possible means. In consequence, a heavy and unhealthy fluctuation raises advertising expenses considerably. Therefore we need a new concept of publicity. The author sees a starting point in the parishes themselves. This start could, in his view, be based upon, and incorporated into a new Communications concept for every diocese.

\section{RESUMEN}

En todas las deliberaciones para mejorar la prensa católica en la República Federal Alemana se desestima, la mayoría de las veces, la captación de abonados. Contrariamente a lo que sucede con los semanarios políticos, las revistas ilustradas y otras publicaciones de gran público, la prensa confesional no puede utilizar con éxito la propaganda a domicilio. Ésta se vuelve, por lo demás, cada vez más problemática. Los agentes publicitarios hacen propaganda de todo lo imaginable. Necesitan un éxito somero a cualquier precio y por cualquier medio. La consecuencia de ello, una acusada e insana fluctuación, eleva los costes de la publicidad extraordinariamente. Por ello, opina el autor, es preciso elaborar nuevos conceptos publicitarios. Ve la posibilidad de encontrar un sustituto en la misma comunidad. Este sustituto podría, en su opinión, fundamentarse en un nuevo concepto del trabajo publicístico en cada diócesis. 